\title{
UTILIZAÇÃO DO BRINQUEDO TERAPÊUTICO NA ASSISTÊNCIA À CRIANÇA HOSPITALIZADA
}

\author{
U SING THERAPEUTIC TOYS IN CARE WITH HOSPITALIZED CHILDREN
}

\author{
Cassiana Mendes Bertoncello FONTES \\ Cleide Carolina da Silva Demoro MONDINI² \\ Márcia Cristina AImendros Fernandes MORAES ${ }^{3}$ \\ Maria Irene BACHEGA 4 \\ Natália Patrisi MAXIMINO5
}

\begin{abstract}
RESUM 0: estudo exploratório e descritivo, parte integrante de um projeto de parceria entre o Serviço de Enfermagem e de Terapia Ocupacional no preparo da criança para as cirurgias eletivas no Hospital de Reabilitação de Anomalias Craniofaciais da Universidade de São Paulo. Objetivo: utilizar o brinquedo como recurso terapêutico no alívio das tensões reais e inconscientes da criança em relação à hospital ização. M étodo: Foi construído um instrumento de coleta dos dados em forma de roteiro observacional, e aplicado em dois momentos: o primeiro consiste no dia anterior à real ização da cirurgia e o segundo momento no dia da cirurgia imediatamente antes de sua real ização. Utilizamos a contação de história e a demonstração das intervenções de enfermagem nos brinquedos (bonecos) com equipamentos e materiais comumente utilizados na hospitalização (luvas, aventais cirúrgico, máscara facial e gorro cirúrgico). Resultados: Dentre as 21 variáveis de comportamento observadas, oito obtiveram diferença estatisticamente significativa com teste de M cN emar $(p>0,05)$. Conclusão: brincar interativamente proporciona à criança hospitalizada interagir com o ambiente hospitalar, expressar os seus sentimentos e emoções e provê recursos para a assistência humanizada.
\end{abstract}

PALAVRAS-CHAVE: educação especial; comportamento da criança; criança hospitalizada; terapia através do brinquedo.

ABSTRACT: This is an exploratory and descriptive study that was jointly carried out by Nursing Care and Occupational Therapy as part of a Research Project that intended to prepare children for elective surgery at the University of São Paulo's Hospital de Reabilitação de Anomalias Craniofaciais. Objective: using toys as a therapeutic resource for relieving the child's real and unconscious tensions concerning hospitalization for surgical treatment at the HRAC - USP. Method: 44 children participated in the study. An observation form was used to collect data and it was applied at two separate times: the first time was the day before the surgery was

\footnotetext{
${ }^{1}$ Doutora em Saúde do Adulto pela Escola de Enfermagem da Universidade de São Paulo. Enfermeira e Diretora Técnica do Serviço de Enfermagem do Hospital de Reabilitação de Anomalias Craniofaciais da Universidade de São Paulo (HRAC/ USP), Bauru/ SP. cmbf@usp.br

${ }^{2}$ Doutora em Fissuras Orofaciais e Anomalias Relacionadas pelo HRAC/ USP, Bauru/ SP. Enfermeira da Unidade de Cuidado Semi-intensiva do HRAC/ USP, Bauru/ SP. cmondini@usp.br

${ }^{3}$ Mestre em Ciências da Reabilitação (Distúrbios da Comunicação Humana) pelo HRAC/ USP, Bauru/ SP. Terapeuta Ocupacional do HRAC/ USP, Bauru/ SP.malmendros@uol.com.br

${ }^{4}$ Doutora em Pediatria pela Faculdade de Medicina de Botucatu, Universidade Estadual Paulista “Júlio de Mesquita Filho" (UNESP), Botucatu/ SP. Diretora do Departamento Hospitalar do HRAC/ USP, Bauru/ SP. dahmib@centrinho.usp.br

${ }^{5}$ Graduanda do curso de Enfermagem da Universidade do Sagrado Coração (USC), Bauru/ SP. natalia.maximino@hotmail.com
} 
to take placeand the second on the day of the surgery just beforetheevent. Twenty one variables wereelaborated by the researchers to categorize behaviors regarding hospitalization. The resources used were: storytelling, dramatization and demonstration of nursing interventions with puppets dressed in surgical garb (gloves, surgical gown, mask and cap) with medical equipment commonly used in hospital. Results: of the 21 variables analyzed, 8showed statistically significant differences on the McN emar Test $(p<0.05)$. Conclusion: interactiveplay enables hospitalized children to interact in the hospital environment, so that they can express feelings and emotions and it contributes to humanized hospital assistance.

KEYWORD S: Special Education; Child Behavior; Hospitalized Children; Occupational Therapy; Toy Therapy.

\section{INTRODUÇão}

Esse estudo foi desenvolvido numa parceria entre o Serviço de Terapia Ocupacional e o Serviço de Enfermagem do H ospital de Reabilitação de Anomalias Craniofaciais daUniversidade deSão Paulo (HRAC-USP), instituição quetem como filosofia a humanização no atendimento aos pacientes com anomalias craniofaciais associadas às fissuras labiopalatinas, desde 1967 (GRACIANO; TAVANO; BACHEGA, 2007). O Serviço de Terapia Ocupacional tem como objetivo tornar o ambiente hospitalar prazeroso durante o processo de hospitalização, por meio de atividades recreativas, educacionais e terapêuticas, preservando e demonstrando o respeito ao usuário e valorização da vida humana (MORAES, 2007).

O terapeuta ocupacional (TO) atua no planejamento e organização deespaços, tornando-os mais humanizados epromovendo a melhora da qualidade de vida e das relações interpessoais. O enfermeiro avalia as necessidades de cuidados de enfermagem da criança nas circunstâncias pré-operatórias, propõe intervenções eavalia os resultados obtidos com as intervenções, responsabilizandose pelo planejamento dos cuidados de enfermagem. Dessa maneira, resolvemos implementar um estudo em queinvestigamos a utilização do brinquedo terapêutico no preparo das crianças em relação aos procedimentos realizados no ambiente hospitalar, durante o no pré-operatório de cirurgias eletivas.

As crianças dispõem de recursos limitados para enfrentar situações desconhecidas. É necessário, então, prepará-las para experiências dolorosas, como a cirurgia. O processo doloroso experimentado pelas crianças durante a hospitalização torna-se menos sofrível quando elas brincam e dramatizam a situação. Ao brincar, a criança terá um meio seguro de expressar verbal e não verbalmente suas emoções, preocupações e percepções em relação à experiência de hospitalização, e não irá se sentir impotente diante desta realidade (RIBEIRO, 1986; NEIRA HUERTA, 1996; A N GERAMI-CAMON, 1998; MARTINS et al., 2001; FALEIROS; SADALA; ROCHA, 2002).

Brincar éuma atividade inerenteao comportamento infantil eessencial ao bem-estar da criança, pois colabora efetivamente para o seu desenvolvimento físico/ motor, emocional, mental esocial, além deajudá-la a lidar com a experiência 
e dominar a realidade. Pode ser considerada como fonte de adaptação, e instrumento de formação, manutenção e recuperação da saúde. Assim, como as necessidades do seu desenvolvimento, a necessidade de brincar não para quando a criança adoece ou é hospitalizada (M ARTINS et al., 2001; WHALEY, 1989).

A equipe de saúde pode criar condições em um espaço lúdico onde a realidade vivenciada pela criança, na fase de hospitalização, seja envolvida pelo imaginário, facilitando sua elaboração e aceitação.

A o brincar de faz-de-conta a criança utiliza sua imaginação, memória, percepção e criatividade, para representar a realidade a seu modo, permitindo a sua manifestação no campo da consciência, de forma menos sofrida e melhor elaborada. Quando a criança representa o que está acontecendo consigo por meio do brincar, ela projeta al go pal pável e visível, e quando projeta ela tem condições de sentir, ver e tocar em algo concreto como nas bonecas. A utilização desses recursos cria condições para a criança poder entender e aceitar melhor o que está se passando com ela. O lúdico contribui para um melhor, mais tranquilo e seguro esclarecimento do processo de hospitalização (OLIVEIRA, 2007).

Uma das estratégias que ajudam a criança a compreender eassimilar o procedimento cirúrgico éo uso do brinquedo terapêutico. Entreas diversas formas de comunicação com a criança, o brinquedo mostra-se como uma das mais eficientes, pois proporciona: diversão, relaxamento, diminuição da ansiedade da separação, alívio das tensões, meio de expressar os sentimentos, recuperação mais efetiva, além de uma melhor aceitação ao tratamento e redução dos efeitos traumáticos da hospitalização (NEIRAHUERTA, 1996; FALEIROS; SADALA; ROCHA, 2002).

Existem dois tipos de brinquedo, o normativo e o terapêutico. Atividades espontâneas que levam ao prazer, sem, no entanto, precisar alcançar um objetivo, constituem o brinquedo normativo, e a sala de recreação é o melhor local para desenvolvêlo. Já o brinquedo terapêutico necessita de um profissional para direcionar a criança. É necessário estimulá-la a participar, e o brinquedo tem como meta conduzir a criança, que vivencia a situação de hospitalização, a um bem-estar físico e emocional. O ambiente deve ser previamente preparado com brinquedos, eexistem técnicas espećficas para sua aplicação (LEITE; SHIMO, 2007).

A sessão com brinquedo terapêutico pode ser realizada por qualquer profissional de saúde, para qualquer criança e em qualquer local que seja conveniente e justificável. As sessões não devem ultrapassar de 15 a 45 minutos (MELO; LEITE, 2008).

O brinquedo terapêutico pode ser classificado de três formas: o brinquedo dramático, brinquedo instrucional eo brinquedo capacitador defunções fisiológicas. O primeiro está relacionado àqueleem que criança utiliza os bonecos e matérias hospital ares para exteriorizarem seus sentimentos o que favorece aos profissionais a identificação do que está afligindo a criança, facilitando a 
comunicação entre ela e os profissionais da saúde. O brinquedo instrucional tem a função de preparar a criança para a hospitalização e deve ser levada em conta a faixa etária da criança. Já o brinquedo capacitador das funções fisiológicas tem o objetivo de contribuir com a melhora física da criança, econsisteem desenvolver atividades que facilitem esta condição (LEITE; SHIMO, 2007). Nesse estudo, o brinquedo terapêutico foi utilizad o para fins instrucionais, com intuito de preparar a criança para a cirurgia el etiva no HRAC.

Considerando que brincar é a principal atividade desenvolvida pela criança e que durante o processo de hospital ização ela não deve ser privada dessa atividade, o objetivo desse estudo foi utilizar o brinquedo terapêutico no preparo da criança que será submetida à cirurgia el etiva no período pré-operatório.

\section{MÉTOdo}

O presente estudo utiliza o método exploratório descritivo e foi desenvolvido por meio de observação de pacientes, com idade entre quatro e 12 anos, deambos os sexos, queiriam ser submetidas à cirurgia eletiva equeconstavam na programação cirúrgica. Os pais e/ ou responsáveis acompanharam os pacientes em dois momentos. O primeiro momento foi realizado no dia anterior à cirurgia no anfiteatro da instituição, durante a realização das orientações pré-operatórias em grupo eindividual aos pacientes eseus responsáveis. Essemomento apresentou média de 30 minutos de duração.

O segundo momento foi realizado no Serviço de Educação e Terapia Ocupacional de Recreação do Hospital, que rotineiramente realiza atividades recreativas aos pacientes internados. Enquanto os pacientes que participaram do primeiro momento aguardavam o horário de serem encaminhados à unidade préanestésica, tinham contato com os mesmos brinquedos e poderiam manipular os materiais no cenário lúdico.

Nos cenários dos locais de observação do primeiro e do segundo momento foram utilizados os seguintes brinquedos e materiais: boneca com curativo cirúrgico de fita adesiva em local de lábio superior e com braceletes em membros superiores, berço hospitalar infantil, suporte, frasco e equipo de soro vazio elimpos, máscara egorro cirúrgico, luvas de procedimento, aventais detecido laváveis, de tamanho infantil e da mesma cor utilizada pela equipe da instituição, seringas e scalps limpos e sem agulhas.

Foram utilizados pelos autores a demonstração no cenário lúdico, a contação de história, a dramatização ea observação das crianças manipulando os materiais e brinquedos. Os procedimentos demonstrados foram: realização de curativo cirúrgico labial, demonstração de punção venosa nas bonecas, colocação deroupa cirúrgica - avental, gorro, máscara eluvas; contação dehistória (A pêndice 1) baseada em literatura (MELO; LEITE, 2008), consistindo na ilustração das situações edecuidados pós-operatórios quanto à alimentação, higieneehidratação. 
A dramatização consistiu no estímulo à iniciativa das crianças em vestir a roupa cirúrgica e de realizar os procedimentos nas bonecas com os brinquedos terapêuticos.

Na sistemática de observação, as autoras utilizaram um instrumento de coleta dos dados (A pêndice 2) construído por elas com base em variáveis e aspectos do comportamento infantil (MARTIN Setal., 2001; PIN HEIRO; LOPES, 1993; SANTOS; BORBA; SABA TÉS, 2000; RIBEIRO; SA BATÉS; RIBEIRO, 2001). Foram observadas 21 variáveis comportamentais nas crianças da amostra e quando o comportamento observado estava presente era assinalado "sim" equando ausente "não".

Os resultados parciais apresentados nesseestudo são partedeum Projeto de Pesquisa denominado "O preparo da criança no pré-operatório utilizando brinquedo terapêutico", queobteve parecer favorável do Comitêde Ética ePesquisa em Seres Humanos da Instituição e foi contemplado com Bolsa Institucional do PIBIC/ CN Pq. Os Termos deConsentimento LivreeEsclarecido, assim como o Termo para autorização defotosefilmagem, foram explicados aos responsáveis pelas autoras e, se aceitos e assinados, as crianças eram incluídas no estudo.

\section{Análise Estatística}

Os itens são as variáveis comportamentais observadas e os instantes um e dois são os dois momentos do estudo. As distribuições conjuntas de cada item nos instantes um e dois podem ser apreciados na Tabela 1.

Para comparar a distribuição dos 21 itens nos instantes um e dois, foi utilizado o teste de $\mathrm{M}$ cN emar por meio do qual foram obtidos os valores de $p$. $\mathrm{O}$ teste de M cN emar é um teste de associação, não paramétrico e pode ser aplicado em amostra simples pareada, com escalas nominais de dois valores possíveis (A GRESTI, 1990). Ficaram estabelecidos como estatisticamente significativos oito dos 21 itens avaliados, com $p<0,05$.

\section{Resultados}

Foram observadas 44 crianças, com idade média de nove anos, 54,5\% do sexo masculino e $45,5 \%$ do sexo feminino. Os resultados obtidos são apresentados na Tabela 1.

Das 21 variáveis comportamentais observadas durantea realização do estudo, oito apresentaram alterações entre os dois momentos e serão discutidas: manipula os brinquedos expostos; brinca interativamente expressando suas emoções; realiza as intervenções no brinquedo; faz uso do "faz-de-conta"; negocia com outras crianças para que suas necessidades sejam atendidas; compartilha os brinquedos com outras crianças; é questionador e col oca as roupas cirúrgicas. 
Tabela 1 - Distribuição dos itens nos instantes um e dois e valores de p do teste de McNemar

\begin{tabular}{|c|c|c|c|c|c|c|c|c|c|c|c|}
\hline \multirow{2}{*}{ Itens } & \multicolumn{8}{|c|}{ Item no instante um / Item no instante dois } & \multirow{2}{*}{ Total } & \multirow{2}{*}{$(\%)$} & \multirow{2}{*}{$\mathrm{p}^{*}$} \\
\hline & não/não & $(\%)$ & não/sim & \begin{tabular}{|l|}
$(\%)$ \\
\end{tabular} & sim/não & $(\%)$ & $\operatorname{sim} / \operatorname{sim}$ & $(\%)$ & & & \\
\hline 1 & 4 & (9) & 16 & (36) & 2 & (5) & 22 & (50) & 44 & $(100)$ & 0,002 \\
\hline 2 & 43 & (98) & 1 & (2) & & & & & 44 & (100) & 1,000 \\
\hline 3 & 39 & (89) & 5 & (11) & & & & & 44 & (100) & 0,074 \\
\hline 4 & 4 & (9) & 25 & (57) & 1 & (2) & 14 & (32) & 44 & (100) & $<0,001$ \\
\hline 5 & 8 & (18) & 22 & $(50)$ & & & 14 & (32) & 44 & (100) & $<0,001$ \\
\hline 6 & 5 & (11) & 18 & (41) & & & 21 & (48) & 44 & (100) & $<0,001$ \\
\hline 7 & 16 & (36) & 11 & (25) & & & 17 & (39) & 44 & (100) & $\mathbf{0 , 0 0 3}$ \\
\hline 8 & 15 & (34) & 15 & (34) & & & 14 & (32) & 44 & (100) & $<0,001$ \\
\hline 9 & 13 & (30) & 13 & (30) & 5 & (11) & 13 & (30) & 44 & (100) & 0,099 \\
\hline 10 & 2 & (5) & 1 & (2) & 5 & (11) & 36 & (82) & 44 & (100) & 0,221 \\
\hline 11 & & & 2 & (5) & 5 & (11) & 37 & (84) & 44 & (100) & 0,450 \\
\hline 12 & 1 & (2) & 4 & (9) & 6 & (14) & 33 & (75) & 44 & (100) & 0,752 \\
\hline 13 & 42 & (95) & 2 & (5) & & & & & 44 & (100) & 0,480 \\
\hline 14 & 42 & (95) & 2 & (5) & & & & & 44 & (100) & 0,480 \\
\hline 15 & 38 & (86) & 1 & (2) & 5 & (11) & & & 44 & (100) & 0,221 \\
\hline 16 & 42 & (95) & 2 & (5) & & & & & 44 & (100) & 0,480 \\
\hline 17 & 42 & (95) & 1 & (2) & 1 & (2) & & & 44 & (100) & 1,000 \\
\hline 18 & 3 & (7) & 3 & (7) & 7 & (16) & 31 & (70) & 44 & (100) & 0,343 \\
\hline 19 & 2 & (5) & 3 & (7) & 1 & (2) & 38 & (86) & 44 & (100) & 0,617 \\
\hline 20 & 16 & (36) & 3 & (7) & 15 & (34) & 10 & (23) & 44 & (100) & $\mathbf{0 , 0 1 0}$ \\
\hline 21 & 2 & (5) & 16 & (36) & 4 & (9) & 22 & $(50)$ & 44 & (100) & 0,014 \\
\hline
\end{tabular}

* Teste de McNemar

\section{DıscusSÃo}

Das 44 crianças observadas, 16 não "M anipularam os brinquedos expostos" no primeiro momento, mas manipularam no segundo. Esse aumento na manipulação dos brinquedos ocorreu a partir do momento em que as crianças superavam o primeiro contato com o material exposto, sentindo-se mais à vontade para manipulá-los. 
É importante citar que metade das crianças manipularam os brinquedos nos dois momentos. Esse comportamento é justificado pelo fato de o brinquedo atuar como um efetivo instrumento de comunicação, por meio do qual as crianças podem ouvir explicações dos profissionais e, ao mesmo tempo retirar dúvidas sobre os procedimentos, atenuando efeitos negativos causados pela hospitalização, proporcionando, assim, segurança e conforto ao pequeno paciente (NEIRAHUERTA, 1996; FALEIROS; SADALA; ROCHA, 2002).

Em relação aos comportamentos "brinca interativamente expressando suas emoções" e "realiza as interven ções no brinquedo", houve uma grande variação estatística, pois ao ter contato com o brinquedo no primeiro momento, a maioria das crianças não manifestou interesse em brincar ou real izar al guma intervenção. No entanto, o segundo momento proporcionou uma mai or interação das crianças com os brinquedos e o cenário lúdico. Proporcionou também outra oportunidade para elas exteriorizarem as emoções e conflitos sobre a realidade concreta. A familiarização com os objetos diminui a sensação de estranhamento e temor, ocorrendo uma desmistificação desses objetos (BRANDÃO, 1984; SCHMITZ; PICCOLI; VIERA, 2003).

O resultado encontrado entre os dois momentos do estudo em relação ao comportamento "faz uso do faz-de-conta" demonstra que, ao brincar, a criança utiliza sua imaginação, memória, percepção e criatividade para representar ludicamente no mundo palpável e concreto dos brinquedos os seus medos, incompreensões, fantasias e anseios das circunstâncias vividas. A utilização do brinquedo terapêutico cria condições de a criança poder entender eaceitar mel hor o queestá se passando com ela. O lúdico contribui para um melhor, mais tranquilo e seguro esclarecimento do processo de hospitalização (OLIVEIRA, 2007).

Os comportamentos "negocia com outras crianças para que suas necessidades sejam aten didas" e " compartilha os brinquedos com outras crianças" estavam presentes em grande parte das crianças, porque, de acordo com a idade média das crianças no estudo (9 anos), elas já estão com maturidade para dividir com o outro, assim como também negociar. Isso sugere uma função significativa no processo dinâmico de desenvolvimento da criança, estabelecendo a interação com outras crianças, favorecendo as relações interpessoais e melhorando o estado geral da criança (HORTA, 1989; KUDO; PIERRE, 1990; FRIEDMAN N, 1992; PENTEADO; SEA BRA; PEREIRA, 1996; BORENSTEIN; RIBEIRO; COSTA, 1998; MELLO et al., 1999; HIRSCHHEIMER, 2001).

Quanto ao comportamento "équestionador" , a variação estatística esteve presente tanto nas crianças que não apresentaram esse comportamento nos dois momentos, quanto nas que questionaram apenas no primeiro momento. $\mathrm{O}$ fato de não ocorrerem questionamentos nos dois momentos podeestar rel acionado com o processo de hospitalização, pois no primeiro momento a criança ainda está se adaptando ao contexto hospital ar e no segundo el a encontra-se em uma situação de jejum e pré-cirurgia, o que dificul ta esse tipo de comportamento. 
A maioria das crianças questionou durante o primeiro momento, pois é nesse período que as crianças faziam o contato inicial com os brinquedos terapêuticos, e o próprio cenário lúdico proporcionava um estímulo para sanar possíveis dúvidas referentes ao processo de internação, contribuindo para uma melhor adaptação ao contexto hospitalar.

Para o comportamento "col oca roupas cirúrgicas", metade das crianças vestiram as roupas nos dois momentos. Este dado é justificado pelo fato de as atividades expressivas (teatro, música, dança, fantasias) levarem as crianças a um mundo mágico e cheio de imaginação, no qual podem criar seus personagens e, por meio deles, externar suas emoções (TAKATORI, 2003; AMARAL, 1997).

\section{CONCLUSÃo}

A utilização do brinquedo terapêutico para o preparo pré-operatório de crianças que serão submetidas à cirurgia de correção de fissura labiopalatina podeser considerada como recurso deorientação edeeducação especial no processo de reabilitação dos pacientes no HRAC-USP.

Verificamos a grandeza do estabelecimento da relação terapêutica entreas crianças, profissionais de enfermagem e de terapia ocupacional . A hospitalização poderá se tornar menos traumática e os efeitos negativos poderão ser minimizados se um programa desse modelo for implementado, colaborando com a humanização da assistência ao paciente e sua família.

\section{Agradecimentos}

Agradecemos ao Hospital de Reabilitação de Anomalias Craniofaciais da Universidade de São Paulo e ao CNPq pela oportunidade de desenvolver este estudo e aos pacientes e seus familiares pela contribuição para compreendermos melhor a nossa prática assistencial.

\section{Referências}

A GRESTI, A. Categorical data analysis. New York: Wiley, 1990.

AMARAL, A.M.A. Teatro de animação: da teoria a prática. São Caetano do Sul: A teliê, 1997. AN GERAMI-CAMON, V.A. U rgências psicológicas no hospital. São Paulo: Pioneira, 1998.

BORENSTEIN, M.S.; RIBEIRO, P.; COSTA, R. Lazer e recreação uma unidade de clínica médica: o que pensam e sentem os pacientes participantes. R evista Brasil eira de E nfermagem, Brasília, v. 51, n. 4, p. 493-510, 1998.

BRANDÃ O, S. O desenvolvimento psicomotor da mão. Rio de Janeiro: Enelivros, 1984. p. 63. COTES, C.; HA NA YAMA, M. M enino sorrisos. São Paulo: Thot cognição elinguagem, 2007. 
FALEIROS, F.; SADALA, M.L.A.; ROCHA, E.M . Relacionamento terapêutico com criança no período perioperatório: utilização do brinquedo e da dramatização. Revista da Escola de Enfermagem da U SP, São Paulo, v. 36, n. 1, p. 58-65, 2002.

FRIEDMANN, A. A criança na brinquedoteca. In: FRIEDMANN, A., (Org.). 0 direito de brincar: a brinquedoteca. 2. ed. São Paulo: Scritta 1992. p. 65-73.

GRACIANO, M.I.;TAVANO, L.D.; BACHEGA, M.I. A spectos psicossociais da reabilitação. In: TRINDADE, I.E.; SILVA FILHO, O.G. Fissuras labiopalatinas: uma abordagem interdisciplinar. São Paulo: Editora Santos; 2007. p. 311-313.

HIRSCH HEIMER, M.R. et al. O trabalho da terapia ocupacional na pediatria. R evista Paulista de Pediatria, São Paulo, v. 19, n. 4, p. 187-194, 2001.

HORTA, A.L.M. Influência do tipo deabordagem para injeções intramusculares nas reações de préescolares. Revista da Escola de Enfermagem da USP, São Paulo, v. 23, n. 2, p. 149163, 1989.

KUDO, A .M.; PIERRE, A .S. A terapia ocupacional com crianças hospital izadas. In: KUDO, A.M.; MARCONDES, E., (Coord.). Fisioterapia, fonoaudi ol ogia eterapia ocupacional em pediatria. São Paulo: Savier; 1990. p. 232-45.

LEITE, T.M.; SHIMO, A.K. O brinquedo no hospital: uma análise da produção acadêmica dos enfermeiros brasileiros. Escola A nna N ery R evista de Enfermagem, Rio deJaneiro, v. 11, n. 2, p. 343-350, 2007.

MARTINS, M.R. et al. Protocolo de preparo da criança préescolar para punção venosa, com utilização do brinquedo terapêutico. Revista Latino-A mericana deEnfermagem, Ribeirão Preto, v. 9, n. 2, p. 76-85, 2001.

MELLO, C.O. et al. Brincar no hospital: assunto para discutir e praticar. Psicologia: teoria e pesquisa, Brasília, v. 15, n. 1, p. 65-74, 1999.

MELO, L.L.; LEITE, T.M. O brinquedo terapêutico como facilitador na adesão ao tratamento de diabetes mellitus tipo 1 na infância. P ediatria M oderna, São Paulo, v. 44, n. 3, p. 100-103, 2008.

MORAES, M.C. A influência das atividades expressivas e recreativas em crianças hospitalizadas com fissura labiopal atina: a visão dos familiares. 2007. 83f. Dissertação (M estrado em Distúrbios da Comunicação Humana) - Hospital de Reabilitação de Anomalias Craniofaciais, Universidade de São Paulo, 2007.

NEIRAHUERTA, E.P. Preparo da criança e família para procedimentos cirúrgicos: intervenção da enfermagem. Revista da Escola de Enfermagem da U SP, São Paulo, v. 30, n. 2, p. 340-353, 1996.

OLIVEIRA, V.B. O lúdico na realidade hospitalar. In: VIEGAS, D., (Ed.) Brinquedoteca hospitalar: isto é humanização. Rio de Janeiro: Wak, 2007. p. 27-32.

PENTEADO, R.Z.; SEA BRA, M .N.; PEREIRA, I.M . A ções educativas em saúde da criança: o brincar enquanto recurso para partici pação da família. Revista Brasileira de Crescimento e D esenvolvimento H umano, São Paulo, v. 6, n. 1/ 2, p. 49-56, 1996.

PIN HEIRO, M.C.D.; LOPES, G.T. A influência do brinquedo na humanização da assistência de enfermagem à criança hospital izada. Revista Brasileira de Enfermagem, Brasília, v. 46, n. 2, p. 117-131, abr./ jun. 1993. 
RIBEIRO, C.A. Efeito da utilização do brinquedo terapêutico, pela enfermeira pediatra, sobre 0 comportamento de crian ças recém-hospital izadas. 1986. 156f. Dissertação (Mestrado em Enfermagem Pediátrica) - Escola de Enfermagem, Universidade de São Paulo, 1986.

RIBEIRO, P.J.; SABATÉS, A.L.; RIBEIRO, C.A. Utilização do brinquedo terapêutico, como um instrumento deintervenção de enfermagem, no preparo de crianças submetidas à coleta de sangue. Revista da Escol a de Enfermagem da U SP, São Paulo, v. 35, n. 4, p. 420-428, 2001.

SANTOS, L.M.C.N.; BORBA, R.I.H.; SABATÉS, A.L. A importância do preparo da criança pré-escolar para a injeção intramuscular com o uso do brinquedo. A cta Paulista de Enfermagem, São Paulo, v. 13, n. 2, p. 52-58, 2000.

SCHMITZ, S. M.; PICCOLI, M.; VIERA, C. S. A criança hospitalizada, a cirurgia e o brinquedo terapêutico: uma reflexão para a enfermagem. Rev. Cienc. Cuid. Saúde, Maringá, v. 2, n.1, p. 67-73, 2003.

TAKATORI, M. 0 brincar no cotidiano da criança com deficiência física: reflexões sobrea clínica da terapia ocupacional. São Paulo: Atheneu, 2003.

WHALEY, L.F. Enfermagem pediátrica: el ementos essenciais à intervenção efetiva. 2. ed. Rio de Janeiro: Guanabara, 1989. 


\section{A PÊndice 1}

História utilizada para explicar as intervenções cirúrgicas durante a sessão do brinquedo terapêutico.

Era uma vez uma menininha chamada Lúcia, que nasceu em uma cidade bem pequeninha lá no interior do Pará. A família da Lúcia ficou muito feliz com a sua chegada, mas um pouco preocupada porqueela tinha um cortena boca. O médico explicou que para corrigir esse problema el a precisaria ficar internada no Centrinho efazer uma cirurgia. Quando ele disse isso, a Lúcia pensou: "Ah! Hospital...Que coisa ruim, que tristeza!".

Mas, quando Lúcia chegou ao Centrinho viu que tudo era diferente. O hospital era grande, com muito verde e muitos brinquedos. Logo que ela chegou, uma enfermeira explicou tudo o que ia acontecer durante o período que ela ficaria internada.

Lúcia entendeu que chegaria no dia da cirurgia em jejum ao hospital e que poderia ficar brincando na Recreação enquanto não chegasse a hora dela operar. Um pouco antes de entrar no Centro-Cirúrgico, a enfermeira explicou que ela iria para o Pré-operatório com um acompanhante e lá teria que col ocar o pijaminha do hospital, escovar os dentes, fazer bochecho com anti-séptico, colocar o gorro, tomar o pré-anestésico e deitar na maca para aguardar a cirurgia.

A enfermeira explicou que se ela entrasse acordada no Centro-Cirúrgico, ela não deveria estranhar ao ver os funcionários de roupa azul, máscara, avental, luva e gorro, pois aquele era o uniforme deles. A Lúcia adorou conhecer os materiais utilizados pelos médicos e enfermeiras do Centro-Cirúrgico e pode atése "fantasiar" de médica!

Outros assuntos foram explicados pela enfermeira, como a importância do sorinho, e da alimentação, pois se ela comesse tudo direitinho ela ficaria forte e o sorinho iria embora rapidinho! Lúcia ficou sabendo quando acordasse da cirurgia, iria para o Pós-operatório, e que um acompanhantejá estaria no quarto esperando por ela, afinal, ninguém quer operar eficar sozinho né?

A enfermeira também falou que Lúcia sairia com um curativo na boca e com os braços esticados com um canudo. Mas por quê? O canudo é para ela não colocar a mão na boca, porque senão ela levaria "bichinhos" para a cirurgia.

Outra coisa muito importante que Lúcia aprendeu é que a sua alimentação seria um pouco diferentenas primeiras semanas. O quea Lúcia poderia comer? Coisas líquidas ou pastosas. O que tem no Centrinho pra ela comer? SOPA (tomar sopa nem toda criança gosta, mas a do Centrinho é deliciosa e toda colorida!); LEITE COM CHOCOLATE; GELATINA; IOGURTE; SUCO; SORVETE.

Depois da cirurgia Lúcia dormiu no Pós-operatório e no dia seguinte ela acordou e foi para o refeitório. Depois de tomar o café da manhã, ela já foi brincar normalmente. O médico logo passou para visitá-la e ela recebeu alta para ir para casa.

Pena que o mesmo não aconteceu com uma amiguinha de Lúcia. A Aline acordou meio enjoadinha da cirurgia evomitou um pouco, e por isso não conseguiu comer direito, então precisou ficar no quarto tomando sorinho por mais um tempinho. Mas sabe quenão foi tão ruim assim? É que aqui no Centrinho as crianças que ficam acamadas recebem brinquedos para brincar na cama, enão demorou muito para a A line se recuperar eir embora também. 


\section{Apêndice 2 - Instrumento de coleta de dados}

\begin{tabular}{|c|c|c|c|c|}
\hline \multicolumn{5}{|c|}{ Instrumento de Coleta de Dados } \\
\hline Nome: & \multicolumn{4}{|l|}{ Idade: } \\
\hline Cirurgia: & \multicolumn{4}{|l|}{ RG: } \\
\hline Data $1^{\circ}$ momento ___ & \multicolumn{4}{|c|}{ Data $2^{\circ}$ momento } \\
\hline \multirow[t]{2}{*}{ Observação de Comportamento } & \multicolumn{2}{|c|}{$1^{\circ}$ Momento } & \multicolumn{2}{|c|}{$2^{\circ}$ Momento } \\
\hline & SIM & NÃO & SIM & NÃ̃o \\
\hline \multicolumn{5}{|l|}{1 - Manipula os brinquedos expostos } \\
\hline \multicolumn{5}{|l|}{2 - Tem medo dos brinquedos } \\
\hline \multicolumn{5}{|l|}{3 - Tem medo ao aproximar-se do pesquisador } \\
\hline \multicolumn{5}{|c|}{4 - Brinca interativamente expressando suas emoções } \\
\hline \multicolumn{5}{|l|}{5 - Realiza as intervenções no brinquedo } \\
\hline \multicolumn{5}{|l|}{ 6 - Faz uso do "faz-de-conta" } \\
\hline \multicolumn{5}{|c|}{$\begin{array}{l}7 \text { - Negocia com as outras crianças para que as suas } \\
\text { necessidades sejam atendidas }\end{array}$} \\
\hline \multicolumn{5}{|c|}{8 - Compartilha os brinquedos com outras crianças } \\
\hline \multicolumn{5}{|l|}{ 9- Assume/Demonstra a liderança } \\
\hline \multicolumn{5}{|l|}{10 - Mostra-se seguro } \\
\hline \multicolumn{5}{|l|}{11 - Demonstra alegria } \\
\hline \multicolumn{5}{|l|}{12 - Atitudes verbalizadas } \\
\hline \multicolumn{5}{|c|}{13 - Chora frente à realização do procedimento lúdico } \\
\hline \multicolumn{5}{|l|}{14 - Agarra-se ao cuidador } \\
\hline \multicolumn{5}{|l|}{15 - Apresenta inquietação } \\
\hline \multicolumn{5}{|l|}{16 - Finge não ouvir } \\
\hline \multicolumn{5}{|l|}{17 - Apresenta alteração de humor } \\
\hline \multicolumn{5}{|l|}{18 - Apresenta comportamento verbal } \\
\hline \multicolumn{5}{|l|}{19 - Apresenta comportamento não verbal } \\
\hline \multicolumn{5}{|l|}{20 - É questionador } \\
\hline 21 - Coloca as roupas cirúrgicas & & & & \\
\hline
\end{tabular}

Recebido: 16/ 08/ 2009

Reformulado: 23/ 12/ 2009

Aprovado: 12/ 02/ 2010 